\title{
Discussion of Derivability of Local Residual Stress Level from Magnetic Stray Field Measurement
}

\author{
Marek Augustyniak $^{1,2}$ - Zbigniew Usarek ${ }^{1}$
}

Received: 8 April 2015 / Accepted: 10 June 2015 / Published online: 1 July 2015

(C) The Author(s) 2015. This article is published with open access at Springerlink.com

\begin{abstract}
The NDT procedure dubbed 'metal magnetic memory' method and the related ISO 24497 standard has found wide industrial acceptance in some countries, mainly in Russia and China. The method has been claimed by some researchers (Roskosz and Bieniek in NDT\&E Int 45:55-62, 2012; Wilson et al. in Sens Actuators A 135:381-387, 2007) as having potential for quantitative determination of local residual stress state in engineering structures, at least for some steel grades. This work presents a critical reexamination of a previous important study by Roskosz and Bieniek, who claimed to have found a direct relationship between local residual equivalent stress levels ranging from 0 to 50 $\mathrm{MPa}$, and the stray field gradients in T/P24 steel sample placed in the Earth's ambient magnetic field. We reconstruct their experiment in a magnetic finite element simulation, computing stray magnetic field and its tangential gradients along the axis of the sample. Different combinations of remanent induction and relative magnetic permeability levels have been modeled, and the influence of geometrical discontinuity is quantified. In order to validate magnetic finite element methodology, a new experiment is presented, along with its numerical counterpart. The magnetic finite element method allowed to obtain a good quantitative correlation with well-controlled stray field measurements. It is demonstrated, that the residual stress level of order of $50 \mathrm{MPa}$ is not the only factor, on which the stray field measurement depends. The geometrical discontinuity and the remanent induction contribute to a higher extent to the field amplitudes. Consequently we prove, that a bidirectional correlation between
\end{abstract}

$\bowtie$ Marek Augustyniak

maugustyniak@mif.pg.gda.pl

1 Gdansk University of Technology, 80233 Gdansk, Poland

2 DES ART Ltd, 81366 Gdynia, Poland the magnetic field gradient and local stress levels cannot be determined because of at least three concurrent inseparable factors on which the measured stray field and its spatial gradient depends.

Keywords Metal magnetic memory - Stray magnetic field $\cdot$ Finite element method $\cdot$ Residual stress evaluation

\section{Introduction}

The NDT procedure based on notions of the metal magnetic memory (MMM) or residual lagnetic field (RMF) method has been promoted by its inventor, A. Dubov, since 1994. In 2007 the procedure obtained an international standardization (ISO24997, [1]), and has been taken up commercially, mainly in Russia and China. It is worth noting, that the mentioned ISO standards were compiled by a direct translation of a Russian GOST standard.

The MMM procedure draws attention for at least two reasons:

- it has been applied in various safety-critical components [2-4]

- it has been claimed as capable of solving the essential problem of structural NDT, namely determining a local stress level from a fast and easily interpretable series of measurements in-situ [5-7].

In the literature, strong promotional aspects can be found, especially in the works by Dubov et al [3,6,7]. Dubov insists on a principal difference between MMM and classic stray field measurements (e.g. MFL) [6], to the advantage of the former. Actually, authors presenting a MMM-based qualitative defectoscopy [8,9], exploit the well-known principles of 
flux leakage detection. MMM appears as a mixture of MFL (in its qualitative aspect) and magneto-elastic (Villari) effect, when stress concentrations are looked for. While a potential advantage of MMM consists in the 'passive mode' (i.e. no need to use an external magnetizing device), there is also a serious disadvantage of low signal-to-noise ratio and consequently much lower sensitivity of MMM to small defects. The low signal-to-noise ratio in MMM stems from the relatively low level of the geomagnetic field. The accuracy of the method remains unknown. In particular, Dubov failed to present reliable data on false positive and false negative rates of detection in comparison with other reference techniques.

A serious doubt arises when quantitative applications of MMM are claimed. In the fundamental paper [10] written by Wlasow and Dubov there is an interesting formula, which defines the local stress maximum as a simple function of $K_{\text {max }}, K_{\text {ave }}$ and $\sigma_{m}$ :

$\sigma_{\max } \sim \sqrt{\frac{K_{\max }}{K_{\text {ave }}}} \sigma_{m}$

where: $K_{\max }$ - the maximum value of the gradient of the RMF components in the area of stress concentration, $K_{a v e-}$ the average value of the gradient of the RMF components in the area under examination, $\sigma_{m}$ - the ultimate strength of the material. These parameters, except for $\sigma_{m}$, are also defined in the ISO24997 standard [1]. The location of maximum field gradient is presented as a position of the highest "stress intensity factor", not only due to an applied or residual stress. A defect or another geometrical change in test specimen could as well produce a stress intensity factor under loading.

If the fundamental formula (1) was correct and widely applicable, as claimed by Dubov et al, it would entail a fundamental break-through in NDT. Consequently, it requires a detailed evidence-based examination.

The quantitative use of any magnetic stray-field NDT (either active or passive) relies in the magneto-mechanical phenomena, i.e. a change of local magnetic properties of a solid as a function of stress. The magnetic properties in play are: magnetization $M(\sigma)$, magnetic permeability $\mu(\sigma)$, coercive field $H_{c}(\sigma)$, remanent induction $B_{R}(\sigma)$. All these variables form a general $B-H(\sigma)$ characteristic, defining the dependence of major and minor hysteresis loops on the local stress.

Several authors [11-15] have studied the direct magnetomechanical problem on idealized flat samples, reproducing Villari's experiment for different steel grades. Some empirical relationships can also be found in the pioneering work by Bozorth [16].

The foundation for analytical description of the $B-H(\sigma)$ was laid by Jiles [17], further extended and sometimes referred to as Jiles-Atherton-Sablik model. Although most of MMM researchers refer to Jiles' model, only few of them
[18] emphasize the complex and multi-factorial relationships which require the use of partial differential equations.

Roskosz with his co-authors systematically studies the qualitative and putative quantitative potential of MMM. He notes the complexity of $B-H(\sigma)$ relationship and presents rather complicated patterns of measured magnetic stray field over a sample with a round hole [19]. However, in [2], he makes an unequivocal statement: 'the magnetic metal memory is a nondestructive method with a great potential and is perfect for many applications.' Another pair of works by Roskosz et al contain two contradicting conclusions:

(a) [20] An attempt was made to strictly follow the MMM procedure as described in the ISO24497 standard, and assess local stress level in a dog-bone sample. Inconsistent results were found, and Roskosz states: 'The only way to account for this is to state that either a mistake was made in the research or the methodology of the method is not fully understood, or the methodology itself is wrong. (...) Quantifying the level of stress concentration or the value of stress at the present stage of the development of the method of MMM testing is a disputable and doubtraising issue.'

(b) [5] Dog-bone samples of S235 and T/P24 steel grades are sequentially loaded beyond the yield limit. The residual stresses in the notch region are calculated using structural FEM. An inverse function: $d H(\sigma) / d x$ is plotted and presented as useful for quantitative determination of local stresses.

Facing these contradictory statements, we have formulated the following questions: Could the field gradients measured by Roskosz and Bieniek over S235 and T/P24 steel samples be due to the residual stress alone? Could a reliable inverse function be defined, allowing for deduction of a local residual stress level from the local stray field gradient?

The answer will be based on available literature, new original experimental data and magnetic finite element method (FEM) modeling. It is interesting to note, that the magnetic FEM was rarely if ever used in the context of quantitative MMM-related research. The authors know only of simulations concerning qualitative magnetic defectoscopy [9,21-23]. The only work aiming at defining a quantitative stress-field with magnetic FEM and experiment [24] did not attain the objectives, as declared by its author.

\section{Experimental Set-Ups and Samples}

Two experiments are discussed in this paper. The first, further referred to as 'E1', was performed by Roskosz and Bieniek and described in their paper [5]. Roskosz and Bieniek interpreted their results as a proof of a direct bidirectional 
Fig. 1 Geometry of the sample used in experiment ' $\mathrm{E} 1$ '.

Uniform thickness $\mathrm{t}=2 \mathrm{~mm}$

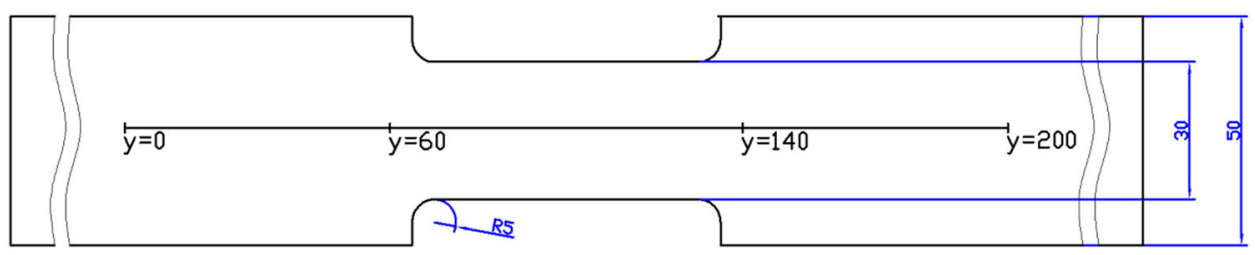

correlation between the local magnetic field gradient and the local stress level in the sample. The second experiment, referred to as 'E2' was performed by the authors of this article, in order to reexamine conclusions put forward by the aforementioned researchers.

\subsection{Reinterpreted Experiment 'E1' by Roskosz and Bieniek}

Roskosz and Bieniek measured two components of static magnetic field along the surface of a flat dog bone-shaped sample. The samples were made of S235 or T/P24 steel grades which were subjected to tensile stress exceeding yield limit and then unloaded. Figure 1 presents the samples' dimensions and the adopted reference coordinate system.

A monotonic and thus potentially useful relationship between maximum magnetic field gradient and local stresses was postulated by those authors. The maximum of $d H / d x$ was shown to be spatially coincident with the maximum of residual stress, produced by tensile loading beyond elastic limit and unloading of the sample. The residual stress was calculated by structural finite element analysis. The most important result was the claimed inverse relationship $\sigma_{e q v} \sim d H / d x$ plotted for T/P24 steel grade within the stress range (0-50 $\mathrm{MPa})$ (Fig. 2).

The experiment by Roskosz and Bieniek had two important features, which influenced both the qualitative and quantitative character of the measurements:

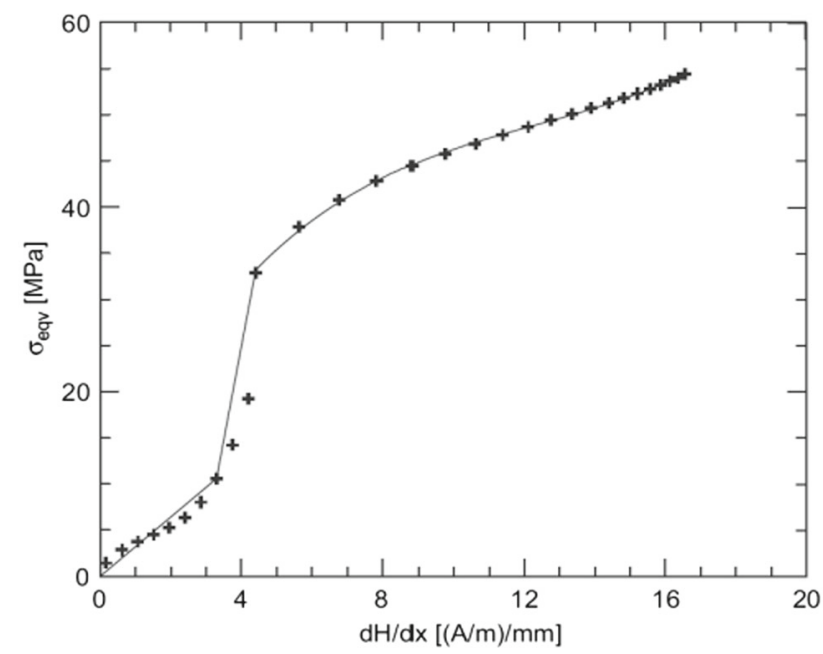

Fig. 2 Inverse relationship $\sigma_{e q v} \sim d H / d x$, steel T/P24 (reprinted from [5]) (a) Standard ferromagnetic clamps of a tensile test machine were used. In literature $[8,25]$ it was shown, that the presence of magnetized clamps may produce an increase of stray field by an order of magnitude, and produce an uncontrollable remanent induction within the sample.

(b) Unlike other authors [8,25], Roskosz and Bieniek extend their measurements over the notched portion of the sample, focusing on the peak found over the geometrical discontinuity. The potential geometrical effect is neglected in their interpretation. However, it can be deduced from the Gauss theorem, and has recently been confirmed experimentally [26,27], that the change of sample width is by itself an important source of stray field, and an enhancement of the local stress intensity factor resulting also in higher residual stress which Roskosz has computed by Finite Element Analysis.

\subsection{New experiment ' $E 2$ '}

In order to resolve concerns about the interpretation of ' $\mathrm{E} 1$ ' results, authors of this paper conducted their own measurements, 'E2'. The samples made of the same material and very similar in shape to those used in experiment ' $\mathrm{E} 1$ ', have been placed in the Earth's magnetic field or alternatively in the controlled field produced by Helmholtz coils. Dimensions of sample 'E2' are shown in Fig. 3. Set-up used in the experiment, which in general enables measurements of stray magnetic field for a variable external magnetic field and loading force, is presented in Fig. 4. In contrast to the experiment 'E1', samples in 'E2' have not been loaded.

The new experiment 'E2' had two objectives. One consisted in assessing qualitatively and quantitatively the stray magnetic field around a sample with a geometric discontinuity, while providing well-controlled initial conditions. The second purpose was to validate the magnetic finite element methodology, comparing simulated results against experimental one.

In order to perform an adequate interpretation of experimental results, FEM magnetostatic modeling in ANSYS software was used. A representative model (corresponding to experiment 'E1') is presented in Fig. 5.

\section{Finite Element Modeling and Its Validation}


Fig. 3 Geometry of the sample used in experiment 'E2'.

Uniform thickness $\mathrm{t}=5 \mathrm{~mm}$

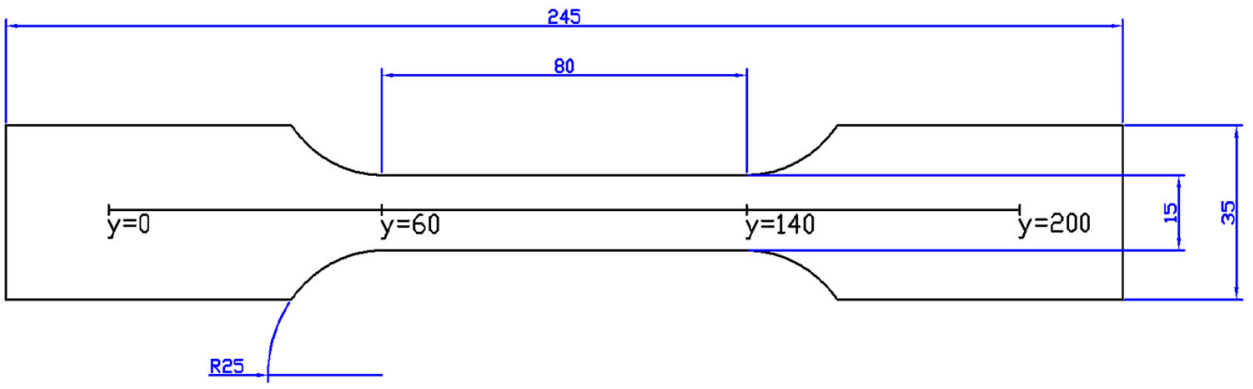

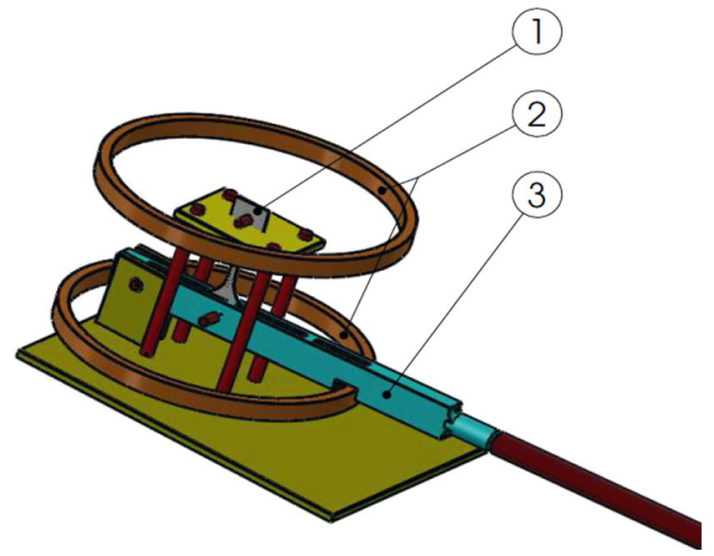

Fig. 4 Diagram of apparatus used in experiment 'E2'. 1—sample, 2Helmholtz coils, 3-tensile machine made of austenitic steel (not used in the experiment)

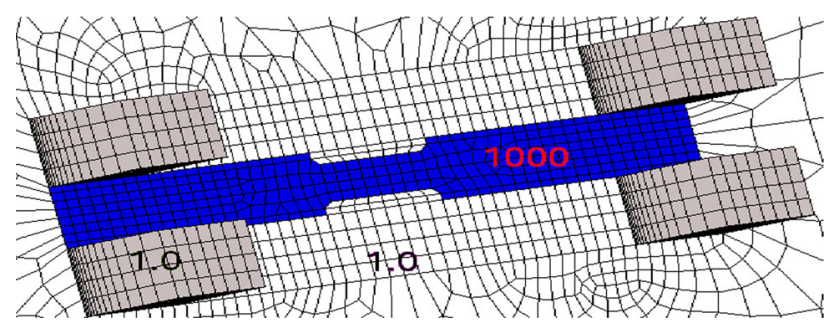

Fig. 5 FEM model of the reference sample in experimental series 'E1'. Numbers indicate typical values of the relative magnetic permeability of each material. Four blocks represent the clamps of the tensile test machine

Dimensions defined in the 3D FEM model are the same as in the experiment 'E1' by Roskosz and Bieniek. Modeled sample is surrounded by solid finite elements representing air. Ambient magnetic flux density in the reference case is equal to $40 \mu \mathrm{T}$ (or $\sim 35 \mathrm{~A} / \mathrm{m}$ in terms of magnetic field strength), a value typical for the tangential component of the Earth's magnetic field. Presence of a ferromagnetic material locally modifies this value according to the Maxwell equations, namely the Gauss's law and Ampere's law. These relationships are compiled by the program into the matrix form and solved using sparse direct algorithm. Although there are four clamps in the model, in presented simulations they are inactive and have properties similar to the surrounding air. Magnetic parameters of the sample, namely its remanence and relative permeability, are variables. The critical region of the model, i.e. a $10 \mathrm{~mm}$ thick air space just above the sample, features regular 3D mesh arranged in layers of progressively increasing thickness.

Remanence modeling in ANSYS is possible in a simplified manner, as described below. The algorithm requires entry of magnetization level 'MG' $[\mathrm{A} / \mathrm{m}]$ which has physical meaning of coercive force. Introduction of 'MG' parameter entails the displacement of the $B-H$ curve along the horizontal i.e. $H$ axis, while its shape is preserved. Consequently, any ( $B$, $H$ ) point located on the linear portion of the hysteresis loop can be accurately modeled, provided that the magnetization is monotonic.

It is relevant to demonstrate, that the adopted modeling scheme produces realistic results. A simulation was thus performed on a model representing the 'E2' sample, placed inside the Earth's field. The axial variation of the absolute gradient of $\mathrm{H}$ is shown in Fig. 6. We decided to present the gradient, and not the smooth $\mathrm{H}(\mathrm{X})$, because the spatial gradient is the critical parameter claim we discuss, opposing the claims of Dubov and others. The gradient is calculated numerically, in an Excel sheet, taking two consecutive points into account (as defined in ISO 24497-2:2007, par. 7.2). Both the experimental and simulated $\mathrm{H}(\mathrm{x})$ functions are relatively smooth, but they contain some noise which becomes apparent on the plot of gradient. In case of simulations, some noise is due to the relative coarse mesh sizing and some assymetry in element shapes between the left and right side of the sample. The measurement is more noisy than the simulation, especially around the left curvature of the sample. These disturbances may be due to the manual finishing of the curved surfaces, and some non-uniform initial state of the material. Both these effects may come about in a real object studied with MFL.

Although there is some discrepancy between measured and computed function around the left peak, a good agreement has been obtained in terms of the maximum of abs. $\operatorname{grad}(\mathrm{H})$, on which the "MMM" procedure relies. This result, together with those from previous works, [28-32] confirms that the magnetic FEM is capable of reproducing space and time field distribution in 3D steel objects of arbitrary shape. 
Fig. 6 Measurement-based (new experiment-'E2') versus computed absolute values of tangential field strength gradient; sample initially demagnetized, unstressed, inside the Earth's field

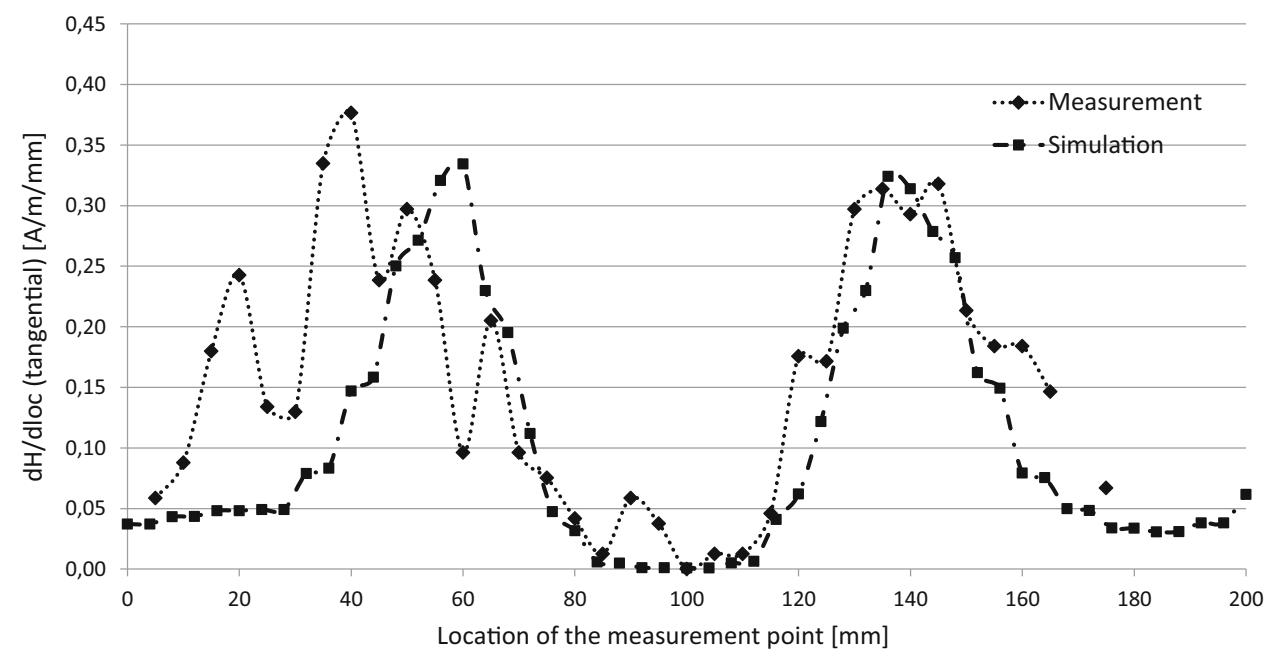

\section{Numerical Sensitivity Analysis of the Rinterpreted Experiment ('E1')}

A sensitivity analysis was performed using magnetostatic FEM. The sample had dimensions reproducing the experiment 'E1' by Roskosz and Bieniek. A linear magnetic permeability of 1000 and a negligible remanent induction $\left(B_{R}\right)$ were assumed as a starting point. The ambient Earth's field strength of $35 \mathrm{~A} / \mathrm{m}$ was defined, in parallel to the sample's axis. Both tangential and normal components of the stray magnetic field were calculated and stored. It was found that the behavior of $d H(\sigma) / d x$ parameter (both axial variation and dependency on studied factors) was similar for $H_{T A N}$ and $H_{N O R M}$, consistently with experimental observations by Roskosz and Bieniek. Consequently, for the sake of succinctness, only sensitivity analysis based on $H_{T A N}$ is presented. The magnetic field strength $H$ variation is shown in Fig 7ac, while the curves in the Fig. $8 \mathrm{a}-\mathrm{c}$ represent the gradient absolute value of $H$, for the same parameters made variable.

\subsection{Sensitivity Analysis of Field Strength Axial Distribution $H(x)$}

The axial variation of the stray magnetic field $\left(H_{T A N}\right)$ depends in a different way on each studied factor $\left(B_{R}, A\right.$, $\left.\mu_{r}\right)$.

The influence of uniform remanent induction $B_{R}$ is nearly linear. Realistic values of $B_{R}(0.2-0.4 \mathrm{~T})$ produce stray field levels exceeding by an order of magnitude the Earth's ambient field. $H_{T A N}$ changes its sign along with the change of sign of $B_{R}$, and the axial variation of $H_{T A N}$ turns from convex to concave.

The influence of the geometrical discontinuity $(A)$ is slightly nonlinear, yet monotonic. Lack of curvature $(A=B)$ produces a nearly constant $H_{T A N}$ along the sample's axis. Increase of $A: B$ ratio results in deepening of the middle min- imum of the curve. However, even the extreme $H$ does not exceed the value of the applied external, ambient Earth's field $(-35 \mathrm{~A} / \mathrm{m})$.

The influence of $\mu$ is notably non-linear and nonmonotonic. There is an extremum of $H_{T A N}$ observed for $\mu \sim 100$. $H_{T A N}$ decreases to nearly zero for $\mu \sim 10,000$, adopting some intermediate levels for $\mu \sim 1000$, which is the typical order of magnitude of initial magnetization of structural steels. Very low $\mu$ makes the sample similar to the surrounding air, and consequently $H_{T A N}$ becomes close to the ambient Earth's field strength $H_{E}$.

\subsection{Sensitivity Analysis of Absolute Value of Field Gradient Axial Distribution $|d H / d x|$}

The absolute value of gradient of $H_{T A N}\left(\left|d H_{T A N} / d x\right|\right)$ adopts a nearly identical, two-peak variation regardless the studied factor, except for the case of $A=B$ (no geometrical discontinuity). The maxima of $\left|d H_{T A N} / d x\right|$ are located over the discontinuities. The highest value of $\left|d H_{T A N} / d x\right|(\sim 4$ $(\mathrm{A} / \mathrm{m}) / \mathrm{mm})$ occurs at $B_{R}=-0.4 \mathrm{~T}$. Without the remanent induction, the maxima of $\left|d H_{T A N} / d x\right|$ reach values about an order of magnitude lower. The largest geometrical discontinuity $(\mathrm{A}=150 \mathrm{~mm})$ produces the maximum of $\left|d H_{T A N} / d x\right|$ equal to $0.7(\mathrm{~A} / \mathrm{m}) / \mathrm{mm}$, and when the initial permeability is variable, then the maximum of $\left|d H_{T A N} / d x\right|(\sim 0.45$ $(\mathrm{A} / \mathrm{m}) / \mathrm{mm})$ is found for $\mu=100$.

\section{Discussion of Results}

The essential question is: could the field gradients measured over S235 and T/P24 steel samples be due to the residual stress alone, as postulated by Roskosz and Bieniek? If that statement was true, then an inverse relationship $\sigma\left(\left|d H_{T A N} / d x\right|\right)$ could indeed be defined, and quantitative, 
Fig. 7 Tangential magnetic field strength along the sample axis for varying: $\mathbf{a}$ uniform remanent induction, $\mathbf{b}$ width of external segments, $\mathbf{c}$ uniform magnetic permeability

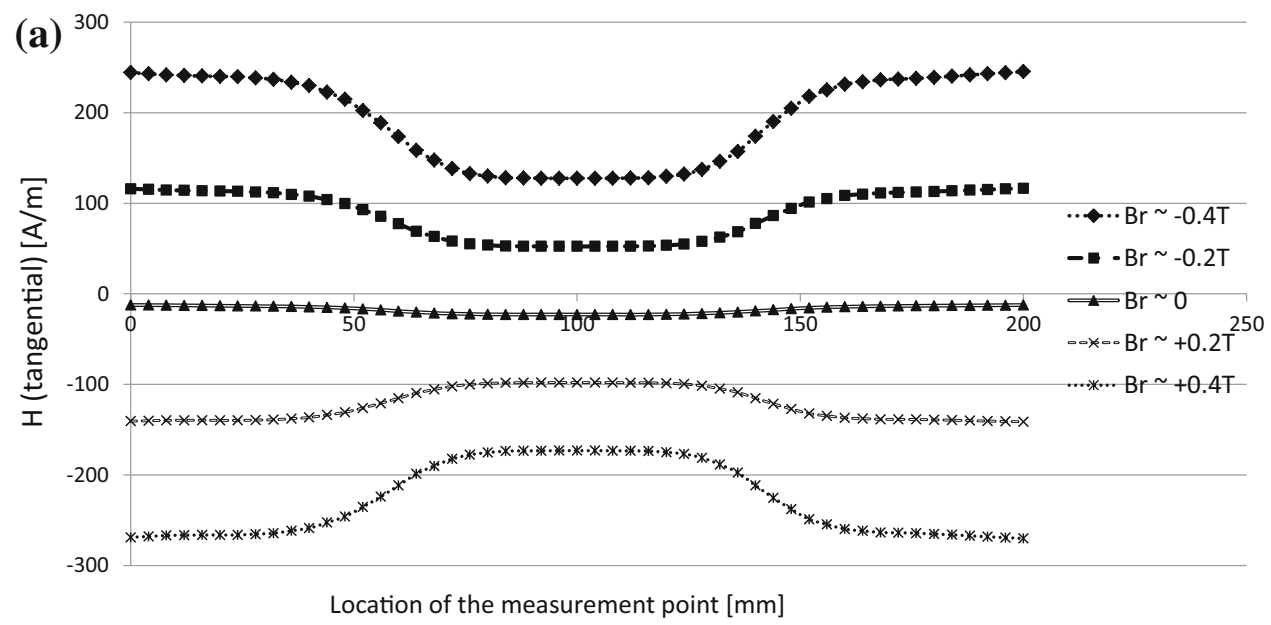

(b)

$\begin{array}{llllll}0 & 50 & 100 & 150 & 200 & 250\end{array}$

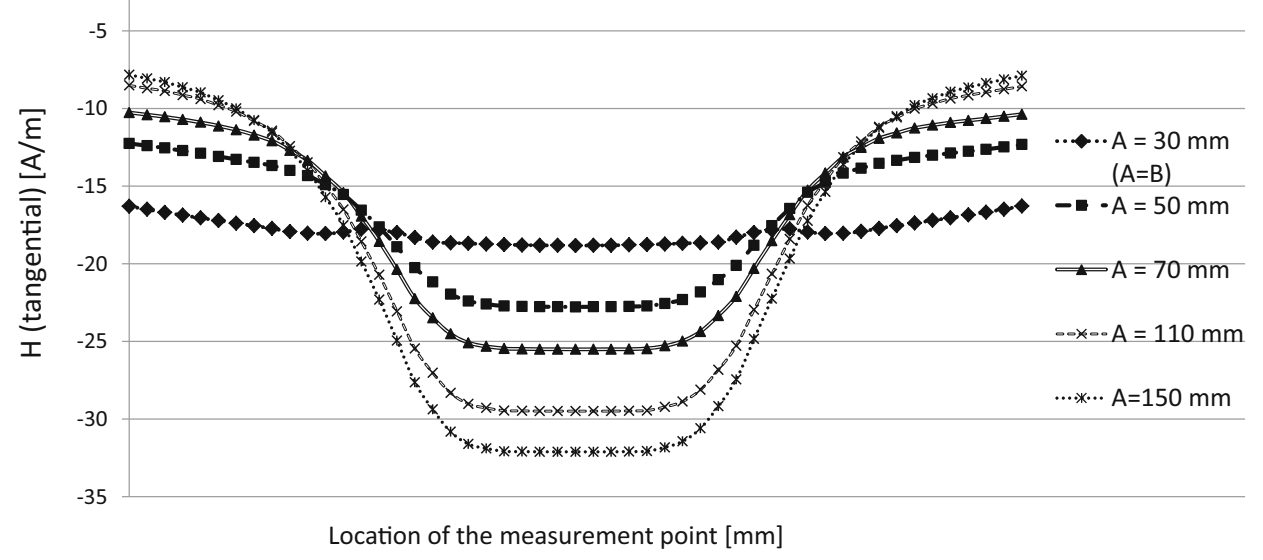

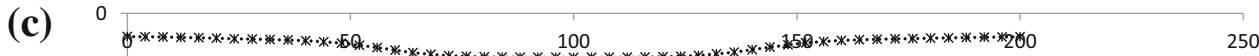

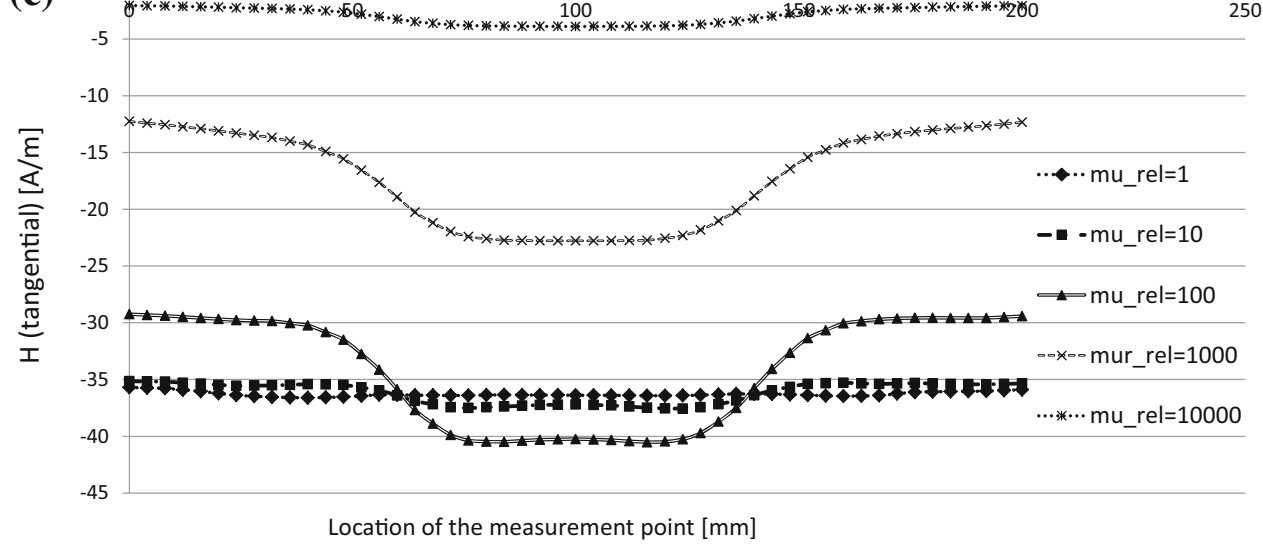

passive static stray field based stress determination would be possible.

Our FEM-based sensitivity analysis along with a consideration of dependencies between structural and magnetic parameters leads to a firm negative answer to the posed question. The main arguments are listed below. (a) The relationship between the material's structural state and the magnetic stray field are non-linear and nonlocal. It is well-known, that the stray field around a ferromagnetic object does not depend in a straightforward manner on the structural stress. Firstly, there is no local-local dependence. Solving Maxwell's equations, 
Fig. 8 Gradient absolute value of tangential magnetic field strength along the sample axis for varying: a uniform remanent induction, $\mathbf{b}$ width of external segments, $\mathbf{c}$ uniform magnetic permeability
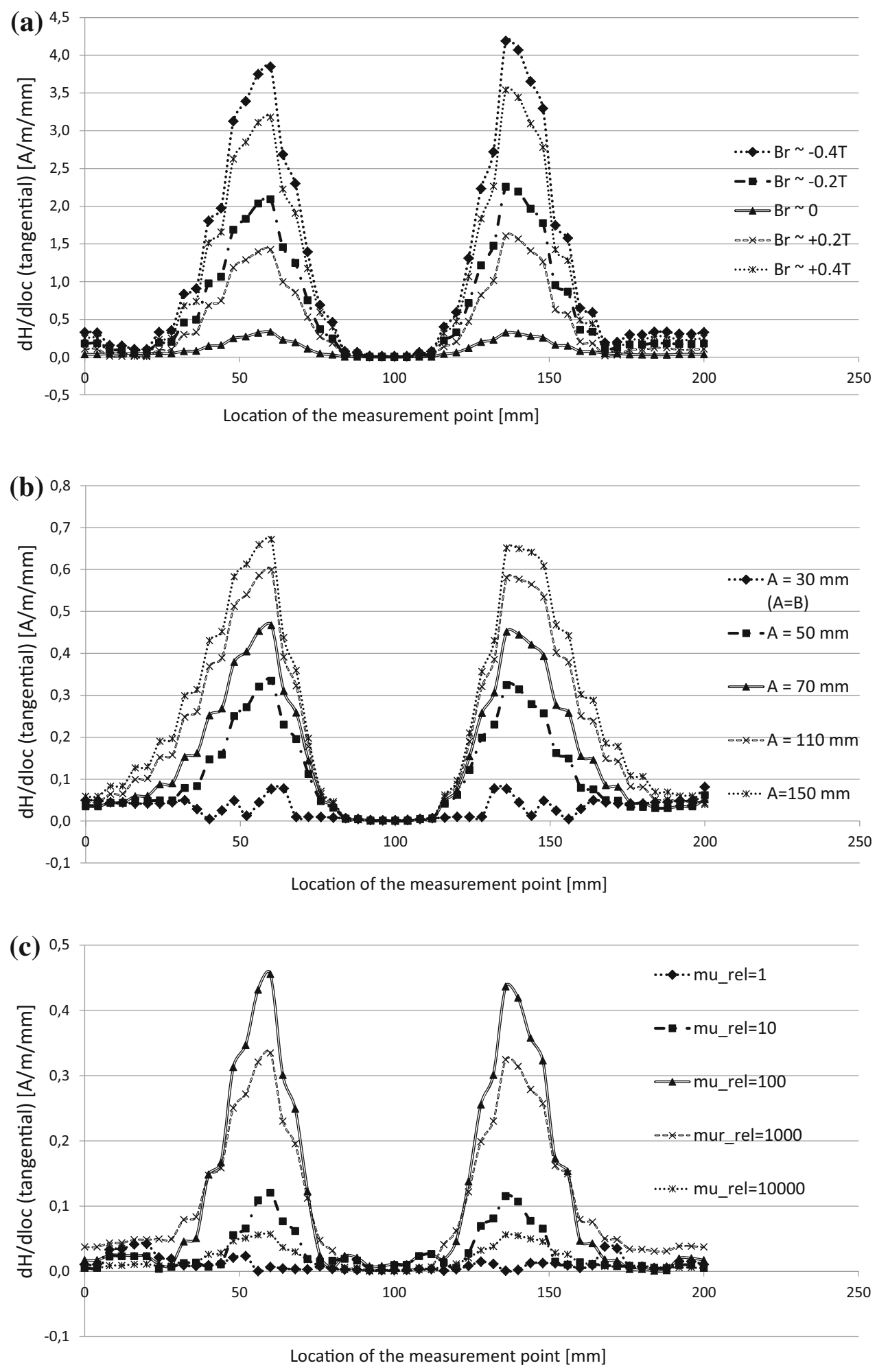

one has to consider an entire magnetic system, composed of various segments of the sample, the ambient air, and possibly other components, such as e.g. the clamps of the tensile test machine. Any change to one of these components, changes the characteristics of the magnetic circuit, thus provoking modification of stray field level and distribution. Secondly, the stress influences more than one local magnetic characteristic of the material, influencing the effective magnetic permeability, remanent induction, and coercivity. Moreover, these 
influences are history-dependent, because a cyclicallymagnetized ferromagnetic material may switch between different hysteresis loops, ranging from the anhysteretic behavior (initial magnetization curve), through minor loops up to the major hysteresis loop [33]. Finally, the residual stress depends on applied load and stress intensity factor as well as the yield strength, which adds further complexity to the problem.

(b) There can be no inverse function of a dependency on several variables. Roskosz and Bieniek draw a relationship $|d H / d x|=f\left(\sigma_{R E S}\right)$, with measured magnetic field gradient and calculated local residual equivalent stress. Further they postulate and define an inverse function, namely $\sigma_{R E S}=f^{-1}(|d H / d x|)$, claimed to be monotonic and valid for T/P24 steel grade. However, as quantified in our sensitivity analysis, the local stray field itself and its gradient are a function of at least three variables, namely the remanent induction $B_{R}$, geometric discontinuity factor $A$, and magnetic permeability $\mu$ :

$H_{L O C A L}=g 1\left(B_{R}\right)+g 2(A)+g 3\left(\mu_{r}\right)$

Furthermore, each of these functions can be separated into a stress-dependent, and a non-stress-dependent contribution, and the presented simple arithmetic sum is only a symbolic representation of a much more complex interdependence between the variables.

(c) Localized stresses of order of $50 \mathrm{MPa}$ in a demagnetized sample cannot produce measurable variation of the stray field. This fact was reported by [8], who gave an explanation derived from Jiles' theory. Our sensitivity analysis corroborates that statement. Based on studies by Anglada and Zurek $[11,24]$ we estimated that the uniform static stress of $\sim 50 \mathrm{MPa}$ along with plastic strain of $\sim 30 \%$ can produce ca $50 \%$ decrease in effective magnetic permeability and $\sim 50 \%$ increase of remanent induction. The effect of localized stress and yield should be even smaller. This variation is not sufficient to produce stray fields and field gradients reported by [5]. Stray field levels measured by Roskosz and Bieniek are of order of 100-200 A/m, and absolute gradients of $H$ reach $5(\mathrm{~A} / \mathrm{m}) / \mathrm{mm}(\mathrm{S} 235$ steel grade) and $20(\mathrm{~A} / \mathrm{m}) / \mathrm{mm}$ (T/P24 steel grade). Comparing these values to those obtained in FEM-based sensitivity analysis one finds, that only an elevated residual induction exceeding 0.4 $\mathrm{T}$ could account for so high magnetic field strength. Indeed, the clamps of the tensile test machine were ferromagnetic, and their remanent induction was neither eliminated nor evaluated during the experiments by Roskosz and Bieniek. During sequential loading, the sample could acquire high, uncontrolled residual magnetization, being at origin of $H$ levels exceeding 100 $\mathrm{A} / \mathrm{m}$. (d) The same value of local $|d H / d x|$ may correspond to different local/global stress states. The FEM-based sensitivity analysis shows, that using the $|d H / d x|$ parameter to determine localized stress levels inevitably leads to ambiguous stress assessment. In the dog-bone sample studied, the axial variation of $|d H / d x|$ is qualitatively identical for all the varied cases, except for a sample without any geometrical discontinuity. Quantification of the $|d H / d x|$ peak does not help either. We found that distinct cases, with possibly various stress levels, resulted in the same maximum of $|d H / d x|$.

\section{Conclusion}

It was found that the stray field gradients reported in the work by Roskosz and Bieniek are quantitatively due to uncontrolled remanent induction. Quantitatively their characteristic two peaks are primarily due to the geometrical discontinuity of the sample.

Several authors present an experimental proof that increasing local stress level or inducing plastic strain makes the strain field varies. However, this does not justify any claim of existence of a single inverse function. The inverse function cannot be defined, mainly because the original magnetic field dependence is a function of several variables. The calculated sensitivity analysis demonstrated the ambiguity of static stray field gradient measurements.

Taking into account our overall experience with electromagnetic NDT, and in view of recently published papers $[26,27]$, the quantitative in-situ NDT based on passive measurement of magnetic stray-field is impossible, despite the attractiveness of its idea.

Open Access This article is distributed under the terms of the Creative Commons Attribution 4.0 International License (http://creativecomm ons.org/licenses/by/4.0/), which permits unrestricted use, distribution, and reproduction in any medium, provided you give appropriate credit to the original author(s) and the source, provide a link to the Creative Commons license, and indicate if changes were made.

\section{References}

1. International Standard "Nondestructive testing-Metal magnetic memory-Part 2: General Requirements", ISO 24497-2:2007

2. Roskosz, M., Rusin, A., Kotowicz, J.: The metal magnetic memory method in the diagnostics of power machinery components. J. Achiev. Mater. Manuf. Eng. 1, 362-370 (2010)

3. Dubov, A., Kawka, A., Juraszek, J.: Application of the metal magnetic memory method for investigation and analysis of stressed states of hoisting mine structure bearing rods. In: Proceedings of ECNDT (2010) Available at, http://www.ndt.net/search/docs. php3? showForm=off\&MainSource $=-1 \&$ KeywordID $=1666$

4. Dubov, A.A.: A technique for monitoring the heating surface tubes of steam and hot-water boilers using the magnetic memory of metals. Therm. Energy 45(1), 59-63 (1998) 
5. Roskosz, M., Bieniek, M.: Evaluation of residual stress in ferromagnetic steels based on residual magnetic field measurements. NDT\&E Int. 45, 55-62 (2012)

6. Dubov, A.: Principal features of metal magnetic memory method and inspection tools as compared to known magnetic NDT methods. In: Proceedings of 16 WCNDT (2004)

7. Tanasienko, A.G., Suntsov, S.I., Dubov, A.A.: Monitoring chemical plant by a metal magnetic memory method. Chem. Pet. Eng. 38, 9-10 (2002)

8. Wilson, J.W., Tian, G.Y., Barrans, S.: Residual magnetic field sensing for stress measurement. Sens. Actuators A 135, 381-387 (2007)

9. Li, Y., Wilson, J., Tian, G.Y.: Experiment and simulation study of 3D magnetic field sensing for magnetic flux leakage defect characterization. NDT\&E Int. 40, 179-184 (2007)

10. Wlasow, W.T., Dubov, A.A.: Assessment of the stress level in concentration zones using Magnetic Memory of Metals (in Polish). In: 14th workshop on NDT, Zakopane (2008)

11. Anglada-Rivera, J., et al.: Magnetic Barkhausen Noise and hysteresis loop in commercial carbon steel: influence of applied tensile stress and grain size. J. Magn. Magn. Mater. 231, 299-306 (2001)

12. Liu, T., Kikuchi, H., Ara, K., Kamada, Y., Takahashi, S.: Magnetomechanical effect of low carbon steel studied by two kinds of magnetic minor hysteresis loops. NDT\&E Int. 39(5), 408-413 (2006)

13. Ossart, F., Hirsinger, L., Billardon, R.: Computation of electromagnetic losses including stress dependence of magnetic hysteresis. J. Magn. Magn. Mater. 196, 924-926 (1999)

14. Dong, L., Xu, B., Dong, S., Song, L., Chen, Q., Wang, D.: Stress dependence of the spontaneous stray field signals of ferromagnetic steel. NDT\&E Int. 42, 323-327 (2009)

15. Iordache, V.E., Hug, E., Buiron, N.: Magnetic behaviour versus tensile deformation mechanisms in a non-oriented Fe-(3 wt\%)Si steel. Mater. Sci. Eng. A 359, 62-74 (2003)

16. Bozorth, R.M.: Ferromagnetism. Wiley-IEEE Press, New York (1993)

17. Jiles, D.C.: Theory of the magnetomechanical effect. J. Phys. D 28, 1537 (1995). doi:10.1088/0022-3727/28/8/001

18. Dapino, M.J., Smith, R.C., Calkins, F.T., Flatau, A.B.: A magnetoelastic model for Villari-effect magnetostrictive sensors. DTIC Report (2002)

19. Roskosz, M., Gawrilenko, P.: Analysis of changes in residual magnetic field in loaded notched samples. NDT\&E Int. 41, 570-576 (2008)

20. Roskosz, M., Bieniek, M.: Analysis of the methodology of the assessment of the technical state of a component in the method of metal magnetic memory testing. In: Proceedings of Defektoskopie 2010/ NDE for Safety, pp 229-236. (2010)
21. Liu, T., Takahashi, S., Kikuchi, H., Ara, K., Kamada, Y.: Stray flux effects on the magnetic hysteresis parameters in NDE of low carbon steel. NDT\&E Int. 39(4), 277-281 (2006)

22. Stupakov, O., Kikuchi, H., Liu, T., Takagi, T.: Applicability of local magnetic measurements. Measurement 42(5), 706-710 (2009)

23. Krause, H.J., Wolf, W., Glaas, W., et al.: SQUID array for magnetic inspection of prestressed concrete bridges. Phys. C 368, 91-95 (2002)

24. Zurek, Z.H.: Magnetic contactless detection of stress distribution and assembly defects in constructional steel element. NDT\&E Int. 38, 589-595 (2005)

25. Leng, J., Xu, M., Zhang, J.: Magnetic field variation induced by cyclic bending stress. NDT\&E Int. 42, 410-414 (2009)

26. Usarek, Z., Augustyniak, B., Augustyniak, M.: Separation of the effects of notch and macro residual stress on the MFL signal characteristics. IEEE Trans. Magn. 50(11), 1-4 (2014)

27. Usarek, Z., Augustyniak, B., Augustyniak, M., Chmielewski, M.: Influence of plastic deformation on stray magnetic field distribution of soft magnetic steel sample. IEEE Trans. Magn. 50(4), (2013)

28. Augustyniak, B., Piotrowski, L., Augustyniak, M., Chmielewski, M., Sablik, M.: Impact of eddy currents on Barkhausen and magnetoacoustic emission intensity in a steel plate magnetized by a c-core electromagnet. J. Magn. Magn. Mater. 272, E543-E545 (2004)

29. Sablik, M., Augustyniak, M.: Nonlinear harmonic amplitudes in air coils above and below a steel plate as a function of tensile strength via finite-element simulation. IEEE Trans. Magn. 40(4), 2182-2184 (2004)

30. Augustyniak, M., Augustyniak, B., Piotrowski, L., Chmielewski, M.: Evaluation by means of magneto-acoustic emission and Barkhausen effect of time and space distribution of magnetic flux density in ferromagnetic plate magnetised by a C-core. J. Magn. Magn. Mater. 304, 552-554 (2006)

31. Augustyniak, M., Augustyniak, B., Sablik, M., Sadowski, W.: The finite element method simulation of the space and time distribution and frequency dependence of the magnetic field, and MAE. IEEE Trans. Magn. 43(6), 2758-2760 (2007)

32. Augustyniak, M., Augustyniak, B., Chmielewski, M., Sadowski, W.: Numerical evaluation of spatial time-varying magnetization of ferritic tubes excited with a C-core magnet. J. Magn. Magn. Mater. 320, 1053-1056 (2008)

33. Dupre, L., Melkebeek, J.: Electromagnetic hysteresis modelling: from material science to finite element analysis of devices. Int. Compumag Soc. Newsl. 10(3), 4-15 (2003) 